\title{
The INTEGRAL Galactic bulge monitoring program: Spectral study
}

\author{
Laura Barragán ${ }^{* \dagger}$ \\ Dr. Karl Remeis-Sternwarte and ECAP, Sternwartstr. 7, 96049 Bamberg, Germany \\ E-mail: laura.barraganesternwarte.uni-erlangen.de
}

\section{Erik Kuulkers, Peter Kretschmar}

European Space Astronomy Centre, SRE-O, 28080 Villanueva de la Cañada (Madrid), Spain

\section{Jörn Wilms}

Dr. Karl Remeis-Sternwarte and ECAP, Sternwartstr. 7, 96049 Bamberg, Germany

\section{Katja Pottschmidt, Anne Lohfink}

CRESST, University of Maryland Baltimore County, 1000 Hilltop Circle, Baltimore, MD 21250, USA

NASA Goddard Space Flight Center, ASD, Code 661, Greenbelt, MD 20771, USA

\begin{abstract}
The center of the Milky Way is a region crowded with X-ray binaries of all kinds: High Mass X-ray Binaries (HMXBs) and Low Mass X-ray Binaries (LMXBs) containing neutron stars or black holes as the compact objects of the binary systems. It then provides a wealth of information on different sources when observed with a wide-field-of-view instrument such as IBIS/ISGRI or JEM-X onboard INTEGRAL. The INTEGRAL Galactic bulge monitoring program was born with the aim of investigating the variability and the transient activity on time scales of days to weeks and months of these sources, in the soft as well as the hard X-ray band [1].

We present here the results of the spectral analysis for eight presistently bright sources in the Galactic bulge that are covered by IBIS/ISGRI and JEM-X simultaneously for the first 3 observing periods (from 2005-02-17 until 2006-03-01), namely 1A 1742-294, 1E 1740.7-2942, 4U 1722-30, GRS 1758-258, GX 3+1, GX 354-0, GX 5-1, and SLX 1744-299/300.
\end{abstract}

8th INTEGRAL Workshop "The Restless Gamma-ray Universe”- Integral2010,

September 27-30, 2010

Dublin Ireland

\footnotetext{
* Speaker.

$\dagger$ We acknowledge support from DLR grant 50OR0701 and ESAC faculty.
} 


\section{Introduction}

The X-ray emission process from LMXBs is thought to depend primarily on the accretion flow structure and its interaction with the compact object. Observations have shown that several sources could be fit with a two component black-body and a disk black-body (diskbb) model ([2, 3]), or with either a single component model, such as a power law or thermal Bremstrahlung ([4] and references therein), or two component models involving a harder component and a softer black-body ([5]). Here we fit the spectra with XSPEC ([6]) using constant *TBnew (diskbb+powerlaw). The constant is introduced to take into account the cross-calibration between the instruments. TBnew is the revised version of the TBabs model $^{1}$ [7].

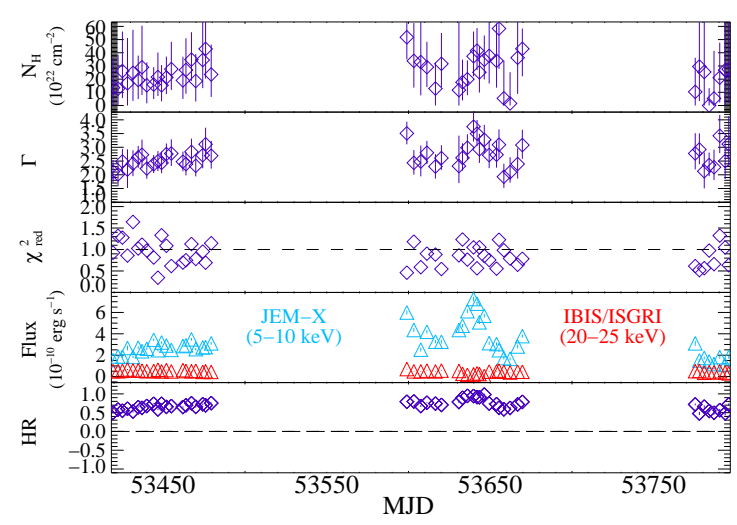

Figure 1: 4U 1722-30 The abundances were set to the interstellar medium abundances summarized by [7]. The fits and the following error determination were done in an automated way making use of a local script. For some sources a unique model was fitted (constant $\star$ TBnew (diskbb+powerlaw)), but for some others the script tried different models, since both of the additive components were not required to provide a good fit; the first model was an absorbed diskbb, and if the $\chi_{\text {red }}^{2}$ was greater than 2 , it was changed to an absorbed power law. If the $\chi_{\text {red }}^{2}>2$ still, the script would fit the complete model (constant $\star$ TBnew (diskbb+powerlaw)).

We studied the evolution of the spectral parameters with time for every source, in a time scale of INTEGRAL revolutions, $\sim 3$ days, although the exposure time (always less than 3 days) varied from revolution to revolution.

For some sources we fixed the hydrogen column $\left(N_{\mathrm{H}}\right)$ to values found in the literature, but for others it was let as a free parameter, since it improved the fit and no clear fixed value was found in our search of the literature. We will focus on the evolution of the $T_{\text {in }}$ parameter of the diskbb model, as a measure of the strength of the soft component; the photon index of the power law $(\Gamma)$; the $\chi_{\text {red }}^{2}$, which is an indicator of the goodness of the fit; the absorbed fluxes for the $5-10 \mathrm{keV}\left(F_{10-15}\right)$ and $20-25 \mathrm{keV}\left(F_{20-25}\right)$ energy ranges, and the hardness ratio (HR), defined as $\frac{F_{10-15}-F_{20-25}}{F_{10-15}+F_{20-25}}$. In a previous analysis, we tried to include a cutoffpl, i.e., a power law with an energy cut-off $\left(E_{\text {cut }}\right)$, but this parameter was not well constrained, and resulted in large uncertainties in the $E_{\text {cut }}$ itself and in the other parameters, therefore it was removed from the fit. This suggests that either the exposure time of every spectrum is not long enough (see the discussion on GRS 1758-258 below) or the cut-off is beyond the studied energy range.

\section{Sources}

In this section we will comment on the main results that we obtain for all the sources. The idea behind using the same model for all of them is to try finding a characteristic diagnostic to differentiate the black hole systems from the neutron star ones.

\footnotetext{
${ }^{1}$ http://pulsar.sternwarte.uni-erlangen.de/wilms/research/tbabs/
} 
4U 1722-30 (Fig. 1) is a binary system containing a neutron star as the compact object, since type-I bursts have been detected at different times (e.g., [1]). This source was well fitted with a power law alone, so we did not include the diskbb component in the XSPEC model. The $N_{\mathrm{H}}$ could not be well constrained. There are changes in the $\Gamma$ and the HR that seem to be correlated with the soft energy flux variations.

SLX 1744-299/300 (Fig. 2) was discovered by Spartan 1 [8]. It consists of two closely spaced objects: SLX 1744-299 and SLX 1744-300, one of them being also a type-I X-ray burster (e.g., [1]). INTEGRAL cannot resolve these two sources, so they are here treated as one. We see that the $N_{\mathrm{H}}$ is not well constrained, being always compatible with a value of few $10^{22} \mathrm{~cm}^{-2}$. The $T_{\text {in }}$ seems to have a constant value of $\sim 2.5 \mathrm{keV}$. The low flux in IBIS/ISGRI is probably causing the poor determination of $\Gamma$.

1A 1742-294 (Fig. 3a) is confirmed to be an X-ray type-I burster since 1994 [9]. In the fit, only one of the additive components (diskbb or powerlaw) was allowed. The $\Gamma$ is relatively soft ( $\sim 2.3)$, and the $T_{\text {in }}$ is around $3 \mathrm{keV}$, except when it goes up to $8 \mathrm{keV}$. This is most probably due to a change in the source (also seen in the HR), which could possibly be better described by a simple power law (the fitting algorithm did not explore this option because the $\chi_{\text {red }}^{2}$ was already good with the diskbb). The $N_{\mathrm{H}}$ is chan-

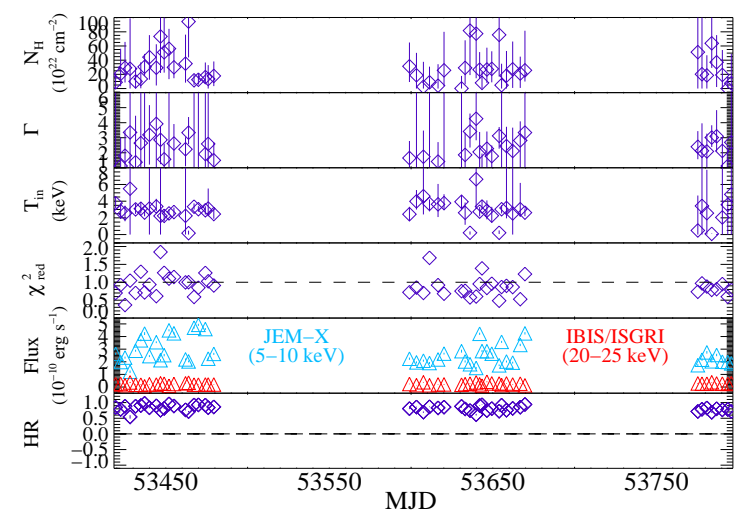

Figure 2: SLX 1744-299/300 ging from being consistent with zero up to some tenths of $10^{22} \mathrm{~cm}^{-2}$. The flux in the softer energy band $(5-10 \mathrm{keV})$ is always greater than the one in the $20-25 \mathrm{keV}$ band.

1E 1740.7-2942 (Fig. 3b) is thought to be a persistent binary black hole [10]. We did not include the diskbb component in the fit, as the power law alone provided a good description of the spectra. The $N_{\mathrm{H}}$ was left free, but it seems to be compatible with a constant value around $25 \times 10^{22} \mathrm{~cm}^{-2}$. We found significant X-ray emission up to $120 \mathrm{keV}$, but we did not detect any energy cut-off as other authors did, probably because our energy range is on the limit of where it was detected, $\sim 100-120 \mathrm{keV}$ ([11]) or the $E_{\text {cut }}$ is even at a higher value ( $\left.>140 \mathrm{keV},[12]\right)$. The source switched off in spring 2006 [1], where the flux was zero in both JEM-X and IBIS/ISGRI instruments. This seems to occur about every 600 days [13]. According to the HR, the source is not changing much, with the flux in the $5-10 \mathrm{keV}$ band being always greater than the one in the $20-25 \mathrm{keV}$ band. However we detect changes in the $\Gamma$, which evolves from 1.6 up to 2.2, in agreement with the values found by [12] ( $\Gamma: 1.52-1.8)$, softer than the ones quoted by [11] $(\Gamma \sim 1.3-1.6)$ or [14] $(\Gamma \sim 1.4)$.

GRS 1758-258 (Fig. 4), is considered to be a microquasar based on its X-ray properties and on the detection of a double-lobed radio counterpart [15]. We wanted to use the same model as we did for the other black hole candidate (BHC) in our sample (1E 1740.7-2942), thus we did not add the diskbb component. Including an energy cut-off resulted in a worse fit, and worse constraints in the parameters, so we kept the description as an absorbed power law, with the $N_{\mathrm{H}}$ fixed to $1.5 \times 10^{22} \mathrm{~cm}^{-2}$ as [16] did, using the value found by [17]. The quality of the spectra above $100 \mathrm{keV}$ is not good, forbidding a good determination of the $E_{\text {cut }}$. However, [18, 19] show 


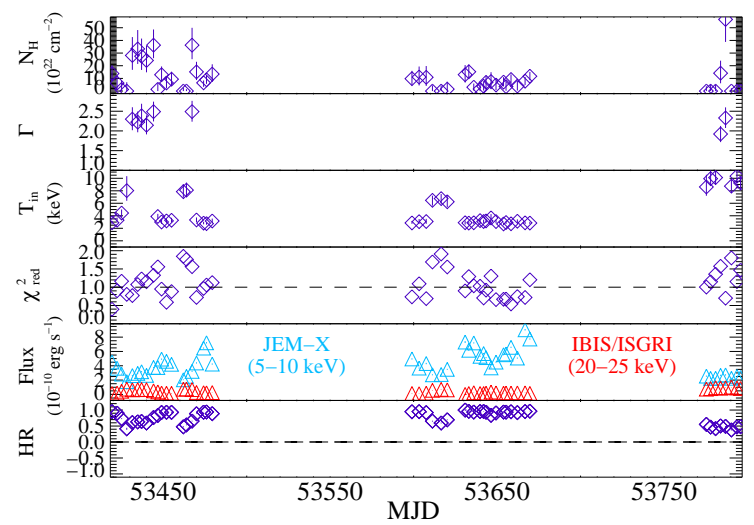

(a) $1 \mathrm{~A} 1742-294$

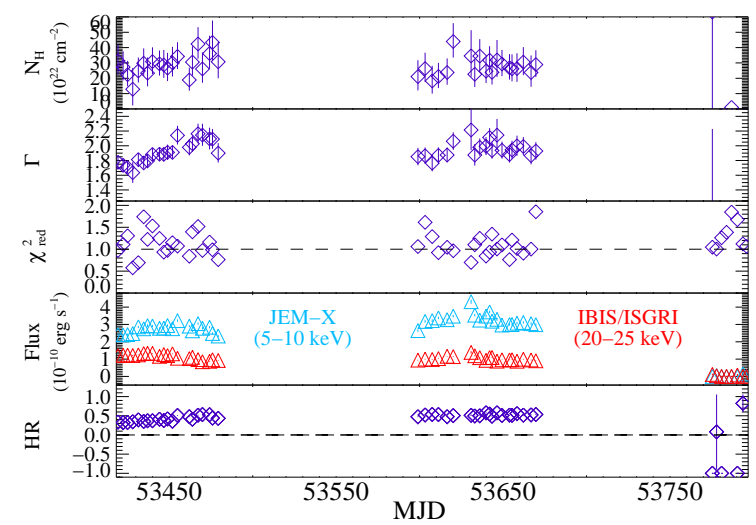

(b) 1E 1740.7-2942

Figure 3

that the cut-off can generally be constrained for this source when the INTEGRAL spectra are averaged over at least a month. Therefore the non-detection of the cut-off is most probably due to too small exposures. In our observation, $\Gamma$ changes from 1.5 to 1.9 , which is what is expected from a canonical hard state of Galactic black hole binaries ([20,21]), and similar to what was found by [16] $(\Gamma \sim 1.54-1.69)$. The $\Gamma$ variations seem to be correlated with the changes in the HR (except for the 3rd period), which points also to a spectral change in the source. See [18] and [19] for an analysis of these datasets modeling spectra averaged over longer timescales.

GX 3+1 (Fig. 5a) is a type-I burster (e.g., [1]) which is very bright in the soft energy band (it has an average flux in the $2-10 \mathrm{keV}$ energy band of half that of the Crab). For our fits we fixed the $N_{\mathrm{H}}$ to $1.6 \times 10^{22} \mathrm{~cm}^{-2}$ as derived by [22]. We can see that the $T_{\text {in }}$ is varying from 2.1 up to 2.5. Since this parameter is related to the emission of the inner edge of the accretion disk, these changes are probably related to variations in the mass accretion rate (e.g., [23]). The $\Gamma$, however, could not be well constrained (it may be due to the low flux in the higher energies), and it is consistent with a constant value around 3 .

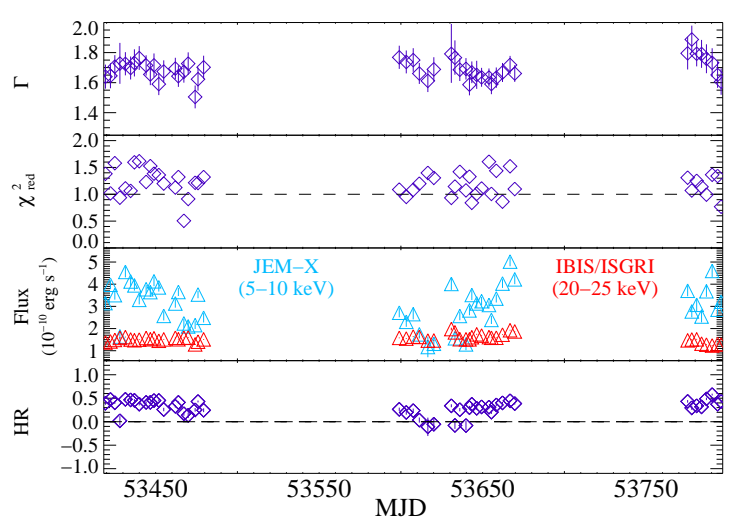

Figure 4: GRS 1758-258

GX 354-0 (Fig. 5b) is a type-I burster (e.g., [1]). The $N_{\mathrm{H}}$ was fixed to $2.9 \times 10^{22} \mathrm{~cm}^{-2}$, as derived in two fits by [24]. They also detected an excess around 6-7 keV, which they attributed to an iron fluorescence line (or lines) coming from the neutral iron in the cool outer disk (and not from the hot inner disk), that can be broadened due to Compton scattering ([25]). The $T_{\text {in }}$ derived from our fits is slightly varying from 2 to $4 \mathrm{keV}$. Sometimes it reaches a higher unphysical value of $\sim 8 \mathrm{keV}$. This can be due to the fitting algorithm not using the right model or it could also be that there is an increase in the $6-7 \mathrm{keV}$ range due to iron fluorescence, which is interpreted by the fit as an increase in the $T_{\mathrm{in}}$. This could well be the case, as most of these times are related to a change in the $5-10 \mathrm{keV}$ flux. We will check this possibility in a future analysis. [24] derived a $\Gamma=1.98$, which is harder than the values obtained from our fits (in general $>2$ ). 


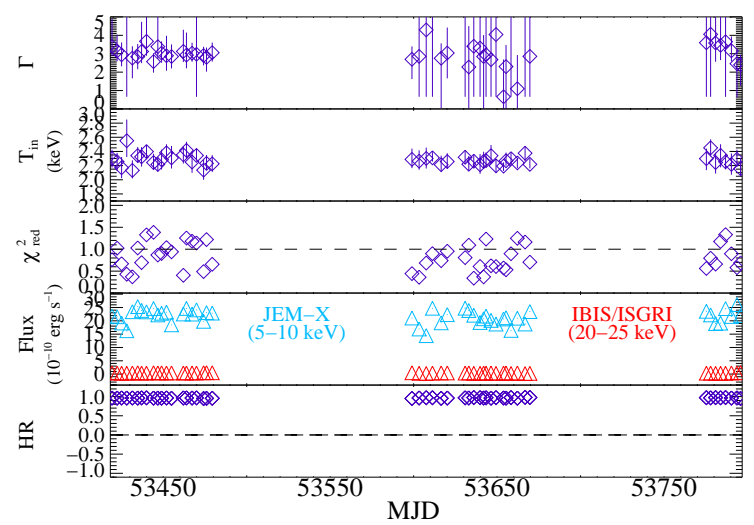

(a) GX $3+1$

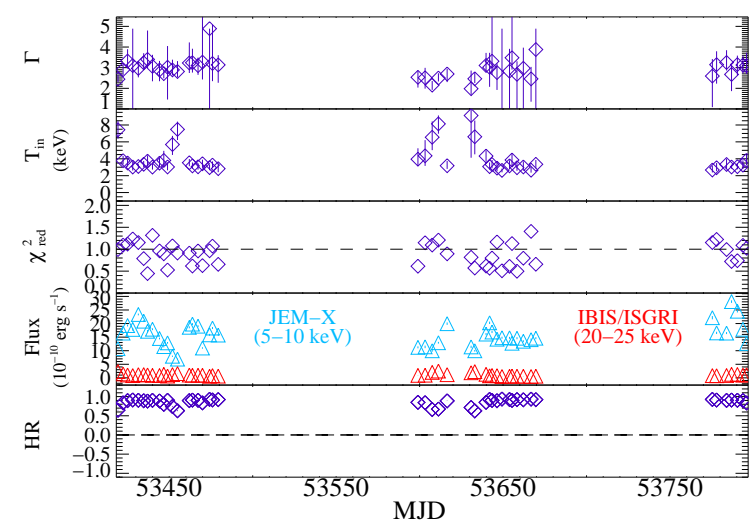

(b) GX 354-0

GX 5-1 is the brightest source in the JEM-X field of view. It is thought to be a Z-source (see [26] and references therein), thus a binary system containing a neutron star. We assumed a fixed $N_{\mathrm{H}}$ of $3 \times 10^{22} \mathrm{~cm}^{-2}$ ([27]). The $T_{\text {in }}$ derived from our fit is well constrained and ranging from 1.7 up to 2.8 . The changes are most probably due to variations in the soft component, since the fluxes in the $5-10 \mathrm{keV}$ band change from 60 to $140 \times 10^{-10} \mathrm{erg} \mathrm{s}^{-1}$. The $\Gamma$ is consistent with a constant value around 3.5 , the uncertainties do

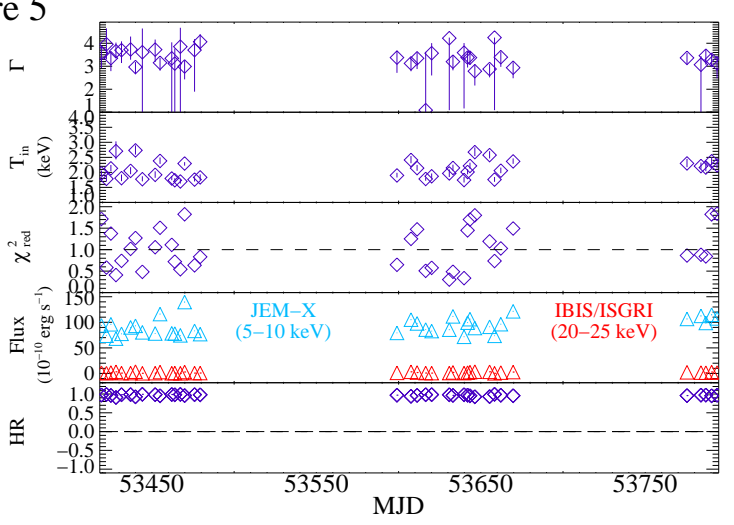

Figure 6: GX 5-1 not allow to claim any evident variation. [28] obtained a $T_{\text {in }}$ ranging from 1.4 to $1.7 \mathrm{keV}$, and they found that the addition of a power law with a $\Gamma$ of 2.5 sometimes helped to improve the fit, which is harder than the one we obtained, although the $T_{\text {in }}$ we find is also higher in general. This could be a correlation among the parameters of the fit (not physical, but mainly due to the mathematical fitting process). It will be checked in our future and more exhaustive work on these sources.

From our analysis of the Galactic bulge monitoring program data presented here, we can say as a preliminary conclusion (more extensive work has to be done), that the black hole binaries seem to have a harder spectrum (i.e. lower value of $\Gamma$ ), as it has been already detected [29] and do not need an additional soft component to describe the data reasonably well. The neutron star binaries, on the other hand, are better described with the addition of a black-body (in our case a diskbb), and have a softer $\Gamma$, in general larger than 2 . No energy cut-off was found in any data set (the parameter was always so poorly constrained that removing it did actually improve the fit). This may be due to not long enough exposures (see discussion about GRS 1758-258) or to the fact that the $E_{\text {cut }}$ can be above of the energy range considered in the fits, and thus not detected.

\section{Outlook}

We intend to carry out a deeper analysis of the data. In the future we want to:

- extend the analysis to all available INTEGRAL data for these sources, analyzing the most recent data and including the public data from other observations to improve the statistics. 
- include all the sources that are observed by both JEM-X and IBIS/ISGRI.

- study the sources that are only visible with one of the INTEGRAL instruments.

- compare the INTEGRAL data with other simultaneous observations with other satellites.

- try different models for every kind of source in order to obtain a better description of the data.

\section{References}

[1] Kuulkers E., Shaw S. E., Pazis A., et al., 2007, A\&A 466, 595

[2] Mitsuda K., Inoue H., Koyama K., et al., 1984, PASJ, 36, 741

[3] White N. E., Stella L., Parmar A. N., 1988, ApJ 324, 363

[4] White N. E., Nagase F., Parmar A. N., 1995, in X-Ray Binaries, ed. W. H. G. Lewin, J. van Paradijs, E. P. J. van den Heuvel (Cambridge: Cambridge Univ. Press)

[5] Callanan P. J., Penny A. J., Charles P. A., 1995, MNRAS 273, 201

[6] Arnaud K. A., 1996, Astronomical Data Analysis Software and Systems V, ed. G. H. Jacoby \& J. Barnes, ASP Conf. Ser. 101, 17

[7] Wilms J., Allen A., McCray R., 2000, ApJ 542, 914

[8] Kawai N., Fenimore E.E, Middleditch J., et al., 1988. ApJ 330, 130

[9] Pavlinsky M.N., Grebenev S.A., Sunyaev R.A., 1994, ApJ 425, 110

[10] Sakano M., Koyama K., Tsuru T., et al. 1998, ApJ 505, 129

[11] Del Santo M., Bazzano A., Zdziarski A. A. et. al., 2005, A\&A 433, 613

[12] Reynolds M. T., Miller J. M., 2010, ApJ 716, 1431

[13] Smith D.M., Heindl W.A., Swank J.H., 2002, ApJ., Lett. 578, L129

[14] Gallo E., Fender R. P., 2002, MNRAS 337, 869

[15] Rodriguez L.F., Mirabel I.F., and Marti J., 1992, ApJ Lett. 401, L15-L18

[16] Pottschmidt K., Chernyakova M., Zdziarski A. A. et al., 2006, A\&A 452, 285

[17] Mereghetti S., Cremonesi D. I., Haardt F., et al., 1997, ApJ 476, 829

[18] Pottschmidt, K., et al., 2008, in Proc. 7th INTEGRAL Workshop, 98

[19] Lohfink, A.M., et al., 2011, ApJ, in prep.

[20] Kuznetsov S. I., Gilfanov M. R., Churazov E. M., et al., 1999, AstL 25, 351

[21] Main, D. S., Smith, D. M., Heindl W. A., et al , 1999, ApJ 525, 901

[22] Oosterbroek T., Barret D., Guainazzi M., et al., 2001, A\&A 366, 138

[23] Barret D., 2001, Advances in Space Research, 28, 307

[24] Narita T., Grindlay J. E., Barret, D., 2001, ApJ 547, 420

[25] Sunyaev R. A., Titarchuk L. G., 1980, A\&A 86, 121

[26] van der Klis M., Jansen F., van Paradijs, J., et al., 1985, Nature 316, 225

[27] Asai K., Dotani T., Mitsuda K., et al., 1994, PASJ 46, 479

[28] Paizis A., Ebisawa K., Tikkanen T., et al., 2005, A\&A 443, 599

[29] Tanaka, Y, 2001, ESO Symposia: Black Holes in Binaries, 141-148 